an excess of certain high-energy electrons.

The model casts doubt on theories

suggesting that reported instrument results indicate signs of dark matter.

For a longer story on this research,

see go.nature.com/FKwzJy

\title{
ATMOSPHERIC PHYSICS
}

\section{Bolt from the blue}

Geophys. Res. Lett. doi:10.1029/2009GL041753 (2010) Gamma rays are bursts of high-energy radiation normally associated with powerful astronomical events, but they have also been observed on Earth. Electrons accelerated in lightning bolts to nearly the speed of light are believed to be behind these 'terrestrial' gamma-ray flashes. But the location of the lightning bolts and their flashes - a key fact needed to figure out how the flashes come about - is not known.

Morris Cohen of Stanford University in California and his colleagues have now built a map showing where lightning bolts and their associated flashes occurred. The researchers used satellite data on gamma-ray flashes, and a global network of verylow-frequency antennas to detect radio waves from lightning. They pinpointed the location of 36 lightning bolts that were associated with flashes and determined which came first. The order of the two events was not always the same.

\section{BIOCHEMISTRY}

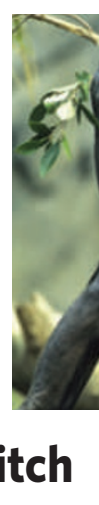

Proc. Natl. Acad. Sci. USA doi:10.1073/ pnas.0911209107 (2010)

The ability to selectively control gene expression has a wealth of applications in biomedical research. Riboswitches are a promising tool; they are a portion of a messenger RNA (which is translated to generate a protein) that can bind to a small molecule to regulate the RNA's activity.

Jason Micklefield and his colleagues at the University of Manchester, UK, have developed riboswitches that, unlike naturally occurring counterparts, respond to synthetic small molecules but not to natural ones. This potentially enables greater gene control.

The authors introduced their switch into Escherichia coli and used it to induce greater gene expression by adding higher concentrations of a synthetic small molecule. They also generated mutated versions of their riboswitches that could induce low, medium or high levels of protein production.

\section{EVOLUTIONARY ANTHROPOLOGY \\ Baby-like bonobos}

Curr. Biol. doi:10.1016/j.cub.2009.11.070 (2010)

Adult bonobos are youthful in play and share food easily. Certain aspects of their behaviour and cognition seem to be developmentally delayed forms of those same traits in the closely related chimpanzee.

To test this hypothesis, Victoria Wobber of Harvard University and her colleagues studied groups of chimpanzees and bonobos of varying ages in the Republic of the Congo and the Democratic Republic of the Congo.

The team shows that whereas chimpanzees (pictured below, right) become less willing to share food as they approach adulthood, bonobos (left) maintain relaxed, juvenile levels of food sharing. Bonobos are also slower to acquire social inhibition. The bonobos' delayed behavioural development correlates with aspects of their cranial morphology, which has also retained juvenile traits. This suggests that these species share a common mechanism responsible for changes in behavioural and brain development.

\section{JOURNAL CLUB}

\author{
Viola Vogel \\ Swiss Federal Institute of \\ Technology, Zurich
}

\section{A bioengineer discusses how mechanical forces in tissues may promote malignancy.}

The connective-tissue protein collagen has been considered to be a structural barrier against tumour invasion in tissues. Enzymes that cleave collagen and other extracellular matrix (ECM) molecules were thus thought to promote tumour progression, but inhibitors of these enzymes have failed in clinical trials. And paradoxically, increased collagen expression is associated with a greater incidence of cancer spread.

Working with mice, Valerie Weaver of the University of California, San Francisco, and her team show that other ECMremodelling parameters regulate malignancy (K. R. Levental et al. Cell 139, 891-906; 2009). They studied an enzyme that initiates collagen crosslinking and is often found in tissue around tumours. They reveal that the crosslinking increases the stiffness of collagen matrices, which upregulates growth-factor signalling and breast malignancy. This suggests that tumour progression depends on a tissue-remodelling process that is regulated by biochemical and mechanical factors.

Bioengineers developing implantable materials that promote tissue regeneration can also learn a lot from this paper. Dense collagen capsules typically form around implanted biomaterials, which has prompted a search for clues to how to engineer surfaces that promote blood-vessel formation and tissue regeneration rather than scarring.

Knowing which factors promote malignancy may also help us to engineer materials and tissues that tip the balance towards enhanced tissue regeneration. This paper might thus stimulate ideas on how to interfere with the interplay between ECM-crosslinking enzymes that enhance matrix stiffness and ECM-protein-cleaving enzymes. Doing so may affect mechanosensitive cell-signalling pathways, promoting regeneration.

Discuss this paper at http://blogs. nature.com/nature/journalclub 